\title{
LAS MEMORIAS DE PRINCIPIOS DEL XIX Y LOS SOUVENIRS DE ÉLISABETH VIGÉE LE BRUN
}

\author{
MEMOIRS OF THE EARLY $19^{\mathrm{TH}}$ CENTURY AND ÉLISABETH \\ VIGÉE LE BRUN'S SOUVENIRS
}

\author{
Mercè BOIXAREU \\ Universidad Nacional de Educación a Distancia \\ mboixareu@flog.uned.es
}

Resumen: El presente estudio aborda la profusión de memorias que se publicaron en la primera mitad del siglo XIX en Francia, así como las razones que subyacen en el desarrollo de este fenómeno. Se ilustrará a través de los Souvenirs de Élisabeth Vigée Le Brun (1755-1842), célebre retratista en la corte de María Antonieta, que se vio obligada a exiliarse durante la Revolución. Entre otras cuestiones, sus Souvenirs dan cuenta de los obstáculos que encontraba como mujer en su labor de pintora, así como de los grandes cambios que presenció: la transición del Antiguo Régimen al Imperio y la Restauración.

Palabras clave: Memorias. Élisabeth Vigée Le Brun. Souvenirs.

Abstract: This study deals with the profusion of memoirs which were published in France in the first half of the $19^{\text {th }}$ century, as well as with the reasons for this phenomenon. This will be illustrated by the Souvenirs of Élisabeth Vigée Le Brun (1755-1842) -a celebrated portraitist in Marie 
Antoinette's court- who was compelled to flee France during the French Revolution. Her Souvenirs are a testimony, amongst other things, of the obstacles she met for being a female painter and of the changes that she witnessed: the transition from the "Ancien Régime" to the Empire and the Restoration.

Key Words: Memoirs. Élisabeth Vigée Le Brun. Souvenirs.

\section{LA PROFUSIÓN DE MEMORIAS EN LA PRIMERA MITAD DEL XIX}

Las memorias de mujeres a principios del siglo XIX están vinculadas a las numerosísimas publicaciones de este género, cerca de 600 de las cuales más de 450 inéditas, que responden indirectamente a un interés lector paralelo. Los estudios de Damien Zanone califican la época que va de 1815 a 1848 como "le grand moment des Mémoires", que corresponde a lo que ha denominado "une fièvre des mémoires", relacionada con un contexto editorial muy favorable (Zanone, 2006: 21-60).

Entre otras razones, para explicar este auge, el autor considera como la más importante e inmediata el hecho de que Francia acababa de atravesar el período más agitado y cambiante de su historia (la Revolución, Napoleón, la Restauración): "les textes des Mémoires apparaissent comme le lieu significatif où s'est exprimé ce temps -c'est-à-dire où l'on a exprimé ce temps" (2006: 18). Durante estos años, cada ciudadano vive su propia y siempre dramática, si no trágica, experiencia y cada autobiografía se convierte en relato de aventuras y supervivencia.

En esta profusión de obras, el número de memorias femeninas guarda proporción con el de las memorias masculinas y la mayoría de las que son anónimas o apócrifas se atribuyen a mujeres (Zanone, 2006: 19).

La literatura memorialista femenina cuenta ya con ilustres precedentes: desde las Memorias de Marguerite de Valois, las de Hortense y Marie Mancini, hasta las más recientes de finales del siglo XVIII, como las de Olympe de Gouges, disfrazada bajo el nombre de Madame de

1. Zanone toma esta expresión del historiador Pierre Nora, al que cita en su estudio (2006: 15).

2. Aquí rescata la expresión de una memorialista: la duchesse d'Abrantès (Zanone, 2006: 23). 
Valmont, o las de Madame Roland, ambas víctimas de la Revolución.

De forma más general, habría que relacionar esta profusión de obras y el éxito del género con lo que hemos denominado la literatura del "yo" en el siglo XVIII, en los distintos géneros literarios y muy especialmente en la narrativa (Boixareu, 2003). Otras causas pueden encontrarse en la cantidad creciente de mujeres lectoras en el siglo XVIII, lo que conllevará un aumento de mujeres escritoras ${ }^{3}$, especialmente a finales del siglo e inicios del siguiente, algunas de las cuales, Madame de Staël, Madame de Genlis, Constance de Salm, nos dejan también sus memorias: las de Madame de Genlis (1825), especialmente influyentes en su tiempo; las de Constance de Salm, menos conocidas, particularmente interesantes por estar compuestas en verso y por sus referencias políticas y literarias ${ }^{4}$.

La importante inclusión de la mujer en la literatura de memorias de esta época responde a dos aspectos de este tipo de literatura. Por una parte, este género se hace eco del devenir histórico, del que algunos personajes eran sus testigos y actores principales. Por esta razón, las memorias habían sido un género reservado a la realeza, a la aristocracia o a los cortesanos, como el cardenal de Retz o Saint Simon, ilustres referentes del género en Francia. Aquí se encuadran también las mencionadas Marguerite de Valois o las Mancini, sobrinas del influyente cardenal Mazarino. Otros textos de autor anónimo o memorias apócrifas pertenecen también a este grupo, como las Mémoires de Christine, reine de Suède (1830), o las Mémoires de Madame la Marquise de Pompadour (1830). De igual forma, la cercanía de Madame de Genlis a la corte y su papel como preceptora de los príncipes de Orléans (el futuro Louis-Philippe) la sitúan dentro de estas memorias de corte. Tras la muerte de la escritora, este monarca ordenará celebrar en su honra funerales solemnes: "À quatre-vingt-quatre ans, la vieille lutteuse avait traversé onze régimes politiques et s'en allait satisfaite de son œuvre" (Trousson, 1996: 777).

Por otra parte, con la Revolución, los actores de la historia dejan de situarse en la corte, y el espíritu republicano convierte en actores a los ciudadanos. Con ello, y a pesar, o trágicamente a causa del olvido de la Revolución por los derechos de las mujeres, muchas de ellas se politizan y sus relatos memorialistas conllevan una fuerte reivindicación feminista,

3. Ver Catriona Seth (2013).

4. Ver Romera Pintor (2015). 
como las ya citadas Olympe de Gouges, Madame Roland o Constance de Salm.

El olvido jurídico y político es paralelo o consecuencia del desprecio a la mujer escritora (que llegará hasta principios del siglo $\mathrm{XX})^{5} \mathrm{y}$, mucho más, a la mujer sobre la cual se escribe: "La gloire d'une femme", según Hortense Mancini (1646-1699), "consiste à ne faire point parler d'elle" (citado por Seth, 2013: 7).

Madame de Genlis recoge y subraya esta misma idea:

Il a fait parler de lui, est toujours un éloge, cela veut dire qu'un homme s'est distingué par ses talents ou ses actions. Elle a fait parler d'elle, est toujours un blâme... Cette phrase signifie que la conduite d'une femme n'est pas irrépréhensible!... Il est donc évident que, pour nous, la véritable gloire ne sera jamais dans la célébrité!... Cela fait rentrer en soi-même (2013: 7).

No obstante, durante las primeras décadas del siglo XIX tenemos ya algunas "célébrités" que nos van a dejar sus memorias, como Madame de Staël, o las de la pintora Vigée Le Brun, en las que nos detendremos más adelante.

En todo caso, este "rentrer en soi-même", propio de las escrituras del "yo", nos lleva a otra dimensión más intimista que se confunde naturalmente con el género de memorias: la escritura autobiográfica, que - con los ilustres precedentes de San Agustín o Santa Teresa- se confirma en Francia con las Confesiones de Rousseau.

Sin entrar en disquisiciones genéricas, ampliamente debatidas y estudiadas $^{6}$, se entiende que la literatura de memorias es fundamentalmente testimonial, e interesa por la particular vivencia de un momento histórico, que nos aparece a partir de la experiencia individual o de forma oblicua a partir del testimonio de un sujeto próximo a la realeza o a sus ministros. Dentro de este grupo podríamos situar, en la época que nos ocupa, las Memorias de Madame de Campan (1822), primera dama de María Antonieta, las Memorias de Madame de Campestre (1827) o las Memorias 
de la duquesa de Abrantès (1831-35), además de las ya citadas de Madame de Genlis.

Otros textos, como el de Madame Roland, se situarían entre las memorias y la autobiografía, por su carácter más introspectivo, incluso de confesión (con alusión explícita a las de Rousseau), pero cuya vinculación con un momento de su tiempo las convierte en un valioso testimonio de la época pre-revolucionaria y de los inicios de la Revolución hasta el Terror.

Además de estos testimonios de una historia del acontecer, las memorias son un valiosísimo documento sociológico y nos descubren las mentalidades de la época. En ellas seguimos, y experimentamos con sus autoras, cómo fue su educación, sus lecturas, las relaciones familiares, el despertar del amor (las más intimistas) y las relaciones con el resto de la sociedad a partir de un estatus determinado.

\section{LOS SOUVENIRS DE ÉLISABETH VIGÉE LE BRUN}

En este contexto de producción, la pintora Élisabeth Vigée Le Brun emprende la redacción de sus memorias ${ }^{7}$, cuya composición se inicia en 1829 con lo que será el primer tomo y se continúa en 1834, con la ayuda de sus sobrinas, Caroline de Rivière y Eugénie Tripier Le Franc. Los dos primeros tomos se publican en 1835, el tercero en 1837. El texto del primer tomo se compone de las cartas dirigidas a la princesa Kourakin, pero a partir del segundo tomo, intitulado Souvenirs, la autora abandona el artificio de la redacción epistolar, aduciendo la muerte de su corresponsal, en 1831. Así lo expresa la memorialista en el "Avant-propos de l'auteur" que abre el segundo tomo:

La mort de la bonne et aimable Princesse Kourakin, que le choléra vint enlever à Pétersbourg en 1831, m'avait fait renoncer pendant longtemps à toute idée de continuer mes Souvenirs, pour lesquels cependant j'avais déjà rassemblé les matériaux nécessaires. Les instances de mes amis m'ayant fait consentir l'an dernier à reprendre ce travail, le lecteur ne sera pas surpris de voir mon second volume

7. En el presente estudio, todas las citas de los Souvenirs de Vigée Le Brun corresponden a la edición de Haroche-Bouzinac de 2008, libro que se reeditará en 2015. 
écrit dans une autre forme que le premier, puisque je n'ai point le bonheur d'achever le récit de ma vie pour celle qui me l'avait fait entreprendre (343).

Biográficamente el relato puede dividirse en una primera parte que corresponde a su vida parisina durante el reinado de Luis XVI y que abarcaría hasta la "Lettre X" del primer tomo, la cual concluye con el triste anuncio del cambio de rumbo que va a afectar su vida con motivo de los primeros sucesos de la Revolución:

Le triste contenu de cette lettre m'avertit que je suis arrivée à l'époque de mon existence dont je voudrais pouvoir perdre la mémoire, dont je repousserais les souvenirs, ainsi que je le fais bien souvent, si je ne vous avais promis le récit sincère et complet de ma vie. Il ne s'agira plus maintenant de joies, de soupers grecs, de comédies, mais de jours d'angoisses et d'effroi et je remets à vous en parler dans mes prochaines lettres. Adieu, chère (239).

Las cartas posteriores, así como el segundo y tercer tomo hasta el capítulo XXVII, comprenden los 12 años de exilio. Desde el capítulo XXVIII se aborda su emotivo retorno a París (el 18 de enero de 1801), sus reencuentros, sus nuevos trabajos como pintora de la sociedad del Imperio, y sus viajes, especialmente a Inglaterra y a los Países Bajos. Un nuevo conjunto de los Souvenirs son las "Lettres" a la condesa Potocka, en las que relata el "Voyage en Suisse en 1808 et 1809". Finalmente, la obra retoma el capítulo XXXIII del tomo III, que se sitúa en la Restauración. Especialmente interesante es este capítulo XXXIII en el que se narra la entrada de Luis XVIII en París: "Enfin Louis XVIII lui-même entra dans Paris, apportant le pardon et l'oubli pour tous" (740).

Entre el tomo I y el II se sitúan sus "Notes et Portraits" sobre los personajes que conoce la autora en la primera época de su vida. Destacan los de Franklin, La Fayette, David o Voltaire, entre tantos otros. Sigue la "Liste des tableaux et portraits que j'avais faits avant de quitter la France en 1789", de la misma forma que entre el tomo II y el III se sitúa otra "Liste de mes portraits". Al final de la obra, la pintora recensa igualmente "Mes portraits faits à Saint Pétersbourg", así como los "Portraits depuis 
mon retour à Paris" $"$ o los "Conseils sur la peinture du portrait".

La autora sobrevive a sus Souvenirs y muere en 1842 bajo la "Monarchie de Juillet". En el "Post-scriptum de l'éditeur", que relata muy sucintamente la vida de la artista y su final ${ }^{9}$, se hace especial hincapié en su talento pictórico y su legado (777). Los motivos que empujan a la memorialista a publicar estos Souvenirs pueden ser diversos. Explícitamente la autora indica que es su amiga la princesa Kourakin la que le incita a escribir su vida, y de hecho, como hemos indicado, la primera parte de esta obra se titula "Lettres à la Princesse Kourakin". No obstante, las formas propias de la literatura epistolar (presente de escritura, interacción con el destinatario) están ausentes de una obra que se inscribe de lleno en la literatura memorialista, sin que la amiga narratoria condicione el relato.

Más allá de esta incitación personal, podemos suponer otras motivaciones de escritura: la voluntad de afirmar su condición de pintora, con una especial reivindicación de independencia en el trabajo y de esfuerzo personal para la consecución de sus éxitos. Esta evidente manifestación de lo que hoy día podríamos llamar "profesionalidad" podría relacionarse con ciertas acusaciones de favoritismo real, como pintora de la reina María Antonieta, así como una supuesta voluntad de desmarcarse de su relación con M. de Calonne ${ }^{10}$.

La sencillez y sinceridad que respira el texto no permite considerar, entre sus motivaciones, la necesidad de "justificar" una vida que, por el tono de la escritura y por sus contenidos, no parece ir más allá de los interesantes "recuerdos" que se evocan. Con todo, sí podría añadirse como motivación el aspecto pecuniario, ya que la autora podría encontrarse en situación de completar sus ingresos con los derivados de la publicación de los Souvenirs.

En lo que a los contenidos de la obra se refiere, toda la primera parte de sus recuerdos viene determinada por su condición de mujer pintora y por las especiales dificultades de su sexo para triunfar.

En su formación artística influyen su padre pintor y la que recibió de sus amigos Doyen y Vernet. A raíz de la muerte del padre y de la posterior boda de la madre, Élisabeth manifiesta su falta de entendimiento con el

8. Sobre las obras de la pintora, ver el catálogo de Joseph Baillio (1982).

9. Sobre los últimos años de su vida, ver Haroche-Bouzinac (2011).

10. Ver la biografía de la autora en Haroche-Bouzinac (2011). 
padrastro, en casa del cual, rue Saint-Honoré, se instala la familia. Es ya en esta época cuando la joven pintora reconoce ser objeto de admiración por su belleza:

Puisque j'ai pris le parti, chère amie, de vous avouer que j'étais toujours remarquée aux promenades, aux spectacles, jusque-là que l'on faisait foule autour de moi, vous devinez sans peine que plusieurs amateurs de ma figure me faisaient peindre la leur, dans l'espoir de parvenir à me plaire; mais j'étais si occupée de mon art, qu'il n'y avait pas moyen de m'en distraire (139).

Como en toda biografía, la autora hace mención de sus lecturas de juventud, relacionadas todas, como era el caso de las mujeres de su época, con obras de piedad, vidas de santos y lecturas apropiadas a una formación según la moral cristiana, con la que la memorialista se muestra acorde:

Puis aussi, les principes de morale et de religion que ma mère m'avait communiqués, me protégeaient fortement contre les séductions dont j'étais entourée. Mon bonheur voulut que je ne connusse pas encore un seul roman. Le primer que j'aie lu (c'était Clarisse Harlove, qui m'avait prodigieusement intéressé), je ne l'ai lu qu'après mon mariage; jusque-là je ne lisais que des livres saints, la morale des saints Pères entre autres, dont je ne me lassais pas, car tout est là, et quelques livres de classe de mon frère (139).

Élisabeth menciona su vinculación con la Académie de Saint Luc, centro corporativo que agrupaba a pintores, escultores y grabadores (y que Turgot disolverá en 1776) y su voluntad y dificultades para ser admitida en la Académie royale (fundada en 1648). Esta Academia celebraba salones de exposición cada dos años y su admisión representaba un signo de reconocimiento como gran artista. En su breve historia (poco más de un siglo), cabe recordar que en 1706 se les cierran las puertas a las mujeres, después de haber admitido sólo a seis. En 1770, cuatro mujeres son aceptadas, de las cuales dos son extranjeras, Rosalba Carriera y madame 
Thersburch ${ }^{11}$. En 1783 Vigée Le Brun postula su admisión, apoyada por Vernet y animada por el éxito de sus cuadros, la mayoría retratos, entre ellos los de la reina María Antonieta. No obstante, y según algunos biógrafos, a pesar del apoyo real, la Academia rechaza la candidatura, puesto que el marido de Élisabeth, Jean-Baptiste Pierre Le Brun, es un conocido marchante ${ }^{12}$. En el rechazo a su candidatura se aduce la voluntad de preservar el arte de su dimensión comercial, dado que el estatus jurídico de la mujer está ligado al del esposo. Finalmente, el 31 de mayo es elegida. En los Souvenirs la pintora relata las intrigas del director, M. Pierre, para impedir su entrada en la institución y el apoyo que recibe de los "vrais amateurs":

Peu de temps après mon retour de Flandre, en 1783, le portrait dont je vous parle et plusieurs autres de mes ouvrages décidèrent Joseph Vernet à me proposer comme membre de l'Académie royale de peinture. M. Pierre, alors premier peintre du Roi, s'y opposait fortement, ne voulant pas, disait-il, que l'on reçût des femmes [...]. Son opposition aurait donc pu me devenir fatale, si dans ce temps-là tous les vrais amateurs n'avaient pas été associés à l'Académie de peinture, et s'ils n'avaient formé, en ma faveur, une cabale contre celle de M. Pierre. [...] Enfin je fus reçue. M. Pierre alors fit courir le bruit que c'était par ordre de la cour qu'on me recevait (180-181).

La artista es recibida en la Academia como "peintre de portrait et d'histoire". La obra de recepción es "La Paix ramenant l'Abondance", realizada en $1780^{13}$. En ella se aprecia y valora especialmente el movimiento que anima a los personajes: la Paz invita a la Abundancia a seguir sus pasos. Los elogios de la crítica son unánimes. Sin embargo, a su regreso del exilio, Élisabeth no podrá volver a la Academia, puesto

11. Ver Linda Nochlin (1993).

12. Ver, en la Introducción de Haroche-Bouzinac, la parte dedicada al capítulo XI, intitulada "L'Académie" (2008: 81-90).

13. En marzo de 2014, este cuadro, propiedad del Louvre desde 1830, se expone de forma destacada como "le tableau du mois n 209 ", elogiando en el folleto que lo acompaña "celle qui fut la plus brillante femme peintre de l'Ancien Régime". 
que las Instituciones post-revolucionarias ya no aceptarán a las mujeres ${ }^{14}$. Es interesante notar que en el discurso - pronunciado con motivo de la creación de la Société populaire et républicaine des Arts en1793- que va a justificar esta exclusión, se menciona a Vigée Lebrun "non pour justifier quelques exceptions", como señala Viennot, "mais pour dénoncer son rôle pervers auprès de la masse des femmes” (2016: 262):

C'est parce qu'une femme célèbre, la citoyenne Lebrun, a montré de grands talents dans la peinture qu'une foule d'autres ont voulu s'occuper de la peinture, tandis qu'elles ne devraient s'occuper que de broder des ceinturons et des bonnets de police.

Durante su exilio, la pintora es recibida con el mayor reconocimiento por parte de los artistas europeos y es admitida en las Academias de Roma, Bolonia, Parma, Berlín, Ginebra, Aviñón y San Petersburgo. La memorialista nos cuenta estos éxitos con simplicidad, espontaneidad y, a menudo, emoción. Se muestra "touchée" -conmovida-y "reconnaissante" -agradecida- frente a las manifestaciones de admiración que recibe: "un artiste travaille toute sa vie pour avoir deux ou trois moments pareils à celui dont je parle" (440).

Vigée Le Brun también hace gala de una profunda libertad de actuación. Relata con gracia sus enfados y sus rechazos, como cuando propone pintar al Papa, pero finalmente decide no hacerlo pues no acepta la obligación que se le impone de cubrirse con un velo para su trabajo.

En los Souvenirs, la autora menciona con gran desenvoltura y sencillez otro aspecto relacionado con su trabajo de pintora: el dinero que gana y que le va a garantizar el reconocimiento social, así como su independencia como mujer, rasgo que caracteriza su vida y su personalidad. En este contexto, explica con manifiesta contrariedad cómo, además de pagar los estudios del hermano, los hombres de su vida disponen de sus ingresos, en primer lugar su padrastro y finalmente, sobre todo, su marido:

M. Le Brun prît dès lors l'habitude de s'emparer des paiements, il n'en imagina pas moins pour augmenter 
notre revenu, de me faire avoir des élèves. Je consentis à ce qu'il désirait, sans prendre le temps d'y réfléchir, et bientôt il me vint plusieurs demoiselles auxquelles je montrais à faire des yeux, des nez, des ovales, qu'il fallait retoucher sans cesse, ce qui me détournait de mon travail et m'ennuyait fortement. [...] Tous ceux qui m'entouraient,

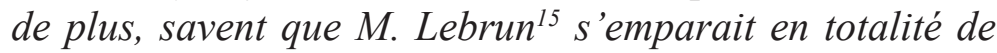
l'argent que je gagnais, me disant qu'il le ferait valoir dans son commerce; je ne gardais souvent que 6 francs de ma poche. Lorsque en 1788 je fis le portrait du beau prince Lubomirski, alors adolescent, sa tante, la princesse Lubomirska, m'envoya douze mille francs, sur lesquels je priai M. Lebrun de me laisser deux louis; mais il me les refusa, prétendant avoir besoin de la somme entière pour solder tout de suite un billet. Il était plus habituel, au reste, que M. Lebrun touchât lui-même, et très souvent il négligeait de me dire que l'on m'avait payée (198).

Esas situaciones la llevan a solicitar la separación de bienes del matrimonio en 1791. Finalmente, se separarán en 1794, cuando ella ya se ha exiliado. Hay que destacar que la memorialista comenta estas circunstancias, se queja, pero no hay indignación ni reivindicación feminista como en otros escritos de mujeres de su época.

Durante el exilio, Vigée Le Brun explica cómo gestiona su dinero a partir de las obras que realiza. Pasa por momentos difíciles, como cuando Napoleón requisiciona el dinero que ella depositó en Venecia, pero siempre los resuelve con trabajo e inteligencia.

Otro aspecto muy importante que narran los Souvenirs es su vida afectiva personal. Si bien no hay relato íntimo en lo que se refiere a su vida sentimental y a sus amores, sí profundiza en su maternidad y en las relaciones con su hija.

En lo referente a la infancia, la memorialista reconoce un afecto especial al padre, que es a la vez su primer maestro y que muere pronto. Cuando la madre se vuelve a casar, Élisabeth no esconde su rechazo al

15. La grafía del apellido del marido alterna a lo largo las memorias: unas veces aparece la forma unida (Lebrun), y otras, la forma separada (Le Brun), tal y como se puede apreciar en el extracto citado. En nuestro estudio empleamos la grafía actual, que separa el artículo del apellido: Le Brun. 
padrastro. Por el contrario, toda su vida permanecerá muy unida al hermano menor.

En cuanto a su matrimonio con el coleccionista y marchante de arte, Le Brun, explica con naturalidad cómo todos los amigos le desaconsejaban tal unión, de forma que se casa en secreto, entre otras razones para no entorpecer una venta. Él no la sigue en su exilio. Se separan poco después, pero es el marido quien facilita, con 120 firmas, que la pintora pueda regresar a Francia.

No obstante, no ofrece ninguna alusión a su vida amorosa, tan sólo un muy indirecto desmentido respecto a su relación con M. de Calonne (199-201). Tampoco se encuentra ni la más mínima alusión a otras posibles relaciones, a pesar de haber sido una mujer que siempre estuvo acompañada y fue admirada por muchos hombres.

Especialmente emotivas son las páginas dedicadas a su hija: su nacimiento, su educación, su distanciamiento doloroso, su muerte. Expresa su alegría cuando se sabe encinta. Uno de los episodios más relevantes, durante su avanzado estado de gestación, es el relacionado con la caída de los pinceles mientras está realizando un retrato de María Antonieta (carta V):

Je me rappelle que dans l'empressement où j'étais de répondre à cette bonté, je saisis ma boîte à couleurs avec tant de vivacité qu'elle se renversa; mes brosses, mes pinceaux tombèrent sur le parquet; je me baissais pour réparer ma maladresse. -Laissez, laissez, dit la reine, vous êtes trop avancée dans votre grossesse pour vous baisser; et, quoi que je pusse dire, elle releva tout elle-même (170).

Este gesto de la reina que se agacha a recoger los pinceles se ha interpretado como un signo de la realeza que se inclina frente al arte. Creemos simplemente que se trata del gesto solidario y atento de una mujer que respeta el estado de la futura madre.

Es interesante también todo el relato en el que, sabiéndose embarazada, la artista continúa con su trabajo hasta el punto de que el parto sobreviene casi en plena actividad:

Ces jouissances d'amour-propre [...] sont bien loin de 
pouvoir se comparer à la jouissance que j'éprouvai lorsque, au bout de deux années de mariage, je devins grosse. Mais ici vous allez voir combien cet extrême amour de mon art me rendait imprévoyante sur les petits détails de la vie; car, tout heureuse que je me sentais, à l'idée de devenir mère, les neuf mois de ma grossesse s'étaient passés sans que j'eusse songé le moins de monde à préparer rien de ce qu'il faut pour une accouchée. Le jour de la naissance de ma fille, je n'ai point quitté mon atelier, et je travaillais à ma Vénus qui lie les ailes de l'Amour, dans les intervalles que me laissaient les douleurs (162).

Élisabeth conjuga a partir de este momento su actividad de pintora con su condición de madre. Esta hija es el gran amor de su vida. Todavía niña, sigue a su madre al exilio y la memorialista relata las congojas relativas a los problemas y dificultades del viaje, la búsqueda y cambio de alojamientos, así como los desvelos por su educación:

[...] car j'avais résolu, tout en courant le monde, de soigner son éducation autant qu'il me serait possible, et je lui avais donné à cet effet à Naples des maîtres d'écriture, de géographie, d'italien, d'anglais et d'allemand. [...] Elle annonçait aussi quelques dispositions pour la peinture; mais sa récréation favorite était de composer des romans. [...] et je me souviens très bien qu'à l'âge de neuf ans elle a écrit à Vienne un petit roman remarquable par les situations autant que par le style (419).

Pasados algunos años, ya en San Petersburgo, la madre explica que desea casarla con un pintor, Guérin, pero la hija se enamora de otro hombre y se casa contra su voluntad, lo que provoca su alejamiento de la ciudad (va a Moscú). Con todo, lo más penoso será la ruptura de las relaciones entre ambas.

Es en el último capítulo cuando Élisabeth nos relata la muerte de su hija como el dolor más profundo de su vida:

Toutefois ce chagrin n'approcha pas de la douleur cruelle 
que me fit éprouver la mort de ma fille. Je m'étais hâtée de courir chez elle, dès que j'avais appris qu'elle était souffrante; mais la maladie marcha rapidement, et je ne saurais exprimer ce que je ressentis lorsque je perdis toute espérance de la sauver: lorsque j'allai la voir, pour le dernier jour, hélas! et que mes yeux se fixèrent sur ce joli visage totalement décomposé, je me trouvai mal [...]. Le lendemain, je n'avais plus d'enfant! [...] Hélas! elle était si jeune! ne devait-elle pas me survivre? (755).

De forma muy sucinta, la autora hace mención del prohijamiento de sus sobrinas, y con ello termina el tercer tomo de los Souvenirs: "Elles me font retrouver tous les sentiments d'une mère [...]. C'est près de ces deux êtres chéris et des amis qui me sont restés que j'espère terminer doucement une vie errante mais calme, laborieuse mais honorable" (763).

En la tradición de la literatura de memorias, el especial interés del texto de Vigée Le Brun consiste en su testimonio de lo que era la nobleza, la "d'épée" y de "robe", y la alta burguesía de los últimos años del Antiguo Régimen. Por su taller pasan y posan para la artista los personajes más notables de su tiempo. Sobre ellos nos ofrece un breve y discreto comentario. Asistimos a banquetes y fiestas diversas que nos son presentadas, no por una aristócrata ociosa, como en otros casos, sino por una mujer que se ha introducido en estos ámbitos gracias a su trabajo de retratista, que le ha otorgado su prestigio reconocido.

Aunque en su texto no aparecen posicionamientos políticos, su cercanía al mundo de la aristocracia y a la reina muy en particular, la obligan a exiliarse en el momento de la Revolución. El 5 de octubre de 1789, Élisabeth parte hacia un exilio que durará doce años:

Les événements de cette journée m'accablaient d'inquiétude sur le sort de Leurs Majestés et sur celui des honnêtes gens, en sorte qu'à minuit, on me traîna à la diligence dans un état qui ne peut se décrire. Je redoutais extrêmement le faubourg Saint-Antoine, que j'allais traverser pour gagner la barrière du Trône. Mon frère, le bon Robert, et mon mari m'accompagnèrent jusqu'à cette barrière, sans quitter un instant la portière de la diligence. Ce faubourg, dont nous 
avions une si grande peur, était d'une tranquillité parfaite; tous ses habitants, ouvriers et autres, avaient été à Versailles chercher la famille royale, et la fatigue du voyage les tenait tous endormis (258).

Además de contarnos las penalidades de su exilio, los distintos viajes y los cambios de ciudad y de residencias, el texto de esta parte de los Souvenirs transmite su visión de artista con la que describe paisajes, amaneceres, puestas de sol, sensible a la luz y al color cambiantes según los momentos del día ${ }^{16}$, pero también según las distintas latitudes en las que va a encontrarse, de Roma a San Petersburgo:

[...] car tous les artistes ont dî sentir comme moi qu'il est impossibledemarcherautourdeRomesanséprouverlebesoin de se servir de ses crayons; je n'ai jamais pu faire un petit voyage, pas même une promenade, sans rapporter quelques croquis. Toute place m'était bonne pour me poser, tout papier me convenait pour faire mon dessin (388).

La visión personal de paisajes se dobla de apuntes y bocetos, y, desde el punto de vista social, de interesantes encuentros con personajes influyentes, como Madame de Staël a la que inmortalizará en un posterior viaje (1808) en su conocido retrato "Corinne au cap Misène", que se conserva en el Museo d'Art et d'Histoire de Ginebra.

En esta parte de la obra, el aspecto testimonial histórico es también de gran importancia, no sólo porque podemos seguir cómo una emigrante francesa sigue y sufre los acontecimientos de su país, las noticias de la muerte de seres conocidos y próximos, sino también porque su proximidad con las cortes europeas la convierte en nuevo testigo privilegiado de otros momentos cruciales. Destacamos entre ellos el interés que ofrece el relato de la muerte de Catalina de Rusia (1796), que la autora vive muy directamente (559-562), y más tarde la relación del complot que termina

16. Ejemplo de su delectación en la descripción paisajística, de exaltación contemplativa, casi mística, es el siguiente extracto de los Souvenirs: "Je ne puis dire la jouissance que j'éprouvais à contempler ces lignes des Apennins jusqu'à l'heure où le soleil couchant les colorait des tons de l'arc-en-ciel! Cette voûte céleste d'un bleu azur, cet air si pur, cette complète solitude, tout m'élevait l'âme; j'adressais au ciel une prière pour la France, pour mes amis, et Dieu sait quel mépris j'éprouvais alors pour les petitesses du monde" (392). 
con la vida de Pablo I en 1801 (612-614).

Con ella seguimos muy indirectamente el paso del Consulado al Imperio de Napoleón y la petición con 120 firmas para que se le permita su regreso a Francia, aprobación que obtiene el 5 de junio de 1800.

\section{CONCLUSIONES}

Los Souvenirs de Vigée Le Brun se inscriben en la tradición testimonial histórica memorialista. Se añade a este interés de reflejo de una sociedad y de una época el mayor interés biográfico de las especiales dificultades de la mujer artista de la época, siempre acompañadas de las propias de una madre. Como hemos indicado, no obstante, el texto de los Souvenirs no cae en lamentaciones, pesimismos, ni en los desgarros que pudieran contenerse en esas circunstancias fatídicas que vivió la autora. La obra, como su vida, respira esfuerzo de superación, serenidad y, a menudo, buen humor y optimismo.

Así pues, los Souvenirs de Vigée Le Brun son el claro ejemplo de la experiencia de una mujer que vive los últimos años del Antiguo Régimen, los inicios de la Revolución, el exilio, el retorno en la época del Imperio y el advenimiento de la Restauración. Su testimonio es especialmente válido por su conocimiento muy directo de la aristocracia y de los reyes, como pintora de corte, especialmente de la reina María Antonieta, así como su posterior relación con las cortes europeas. La autora nos habla también de su vida personal y afectiva. Aunque no nos descubre su intimidad secreta, sí nos participa sus inquietudes, alegrías y desvelos, relacionados con su trabajo de pintora, con su condición de madre, con la sociedad que le rodea.

Entre memorias y autobiografía, el modesto título de Souvenirs indica que es la selección del recuerdo lo que será materia de relato. En él encontramos fundamentalmente la voluntad de la artista, el testimonio valiosísimo de las dificultades que como mujer encuentra en su trabajo y el coraje admirable con el que va superando las especiales y difíciles circunstancias del devenir histórico, todas ellas agravadas a causa de las determinaciones de su sexo.

Élisabeth Vigée Le Brun fue una gran artista, muy valorada en su tiempo y reconocida en la actualidad como una de las mejores pintoras de su época. Sus obras se encuentran en colecciones particulares y en numerosos museos, entre ellos los grandes museos del mundo, el Louvre, 
el Hermitage, el Prado, el Metropolitan.

Siguiendo a Olivier Blanc, la obra pictórica de Vigée Le Brun nos aparece de un realismo positivo y optimista:

Mme Vigée-Lebrun, véritable experte, qui savait tirer profit d'un défaut, métamorphoser des ressemblances par des expressions uniques inspirées de Raphaël qu'elle admirait tant [...] elle prenait des libertés avec la réalité physique autant qu'avec le statut social, prétendait peindre ce qu'elle voyait ou croyait voir et non ce qu'elle savait (Blanc, 2006: $150)$.

Podríamos concluir diciendo que la acción embellecedora que la pintora confiere a sus retratos, de una realidad de la que es muy consciente, la aplicó también a su vida y a sus recuerdos literarios.

\section{REFERENCIAS BIBLIOGRÁFICAS}

BAILliO, J. (1982). Élisabeth Vigée Le Brun, 1755-1842 (Catalogue). Seattle y Londres: University of Washington Press.

BLANC, O. (2006). Portraits des femmes: artistes et modèles à l'époque de Marie Antoinette. París: Didier Carpentier.

BOIXAREU, M. (2003). "El yo en la literatura francesa del siglo XVIII". En Literatura francesa, A. Yllera \& otros (eds.), 143-152. Madrid: UNED.

BONNET. M.-J. (2012). Liberté, Égalité, Exclusion, Femmes peintres en révolution - 1770-1804. París: Vendémiaire.

HAROCHE-BOUZINAC, G. (2008). "Introduction”. En Élisabeth Vigée Lebrun. Souvenirs, 1755-1842, G. Haroche-Bouzinac (ed.), 7-118. París: Éditions Champion.

(2011). Louise Élisabeth Vigée Le Brun, histoire d'un regard. París: Flammarion.

NOCHLIN, L. (1993). Femmes, art et pouvoir. Nîmes: Chambon.

REID, M. (2010). Des femmes en littérature. París: Belin. 
ROMERA PINTOR, Á. M. (2015). "Mes soixante ans y sus referentes políticos y literarios: las memorias en verso de Constance de Salm". Çédille 11, 439-467, https://cedille.webs.ull.es/11-DEF/20romera. pdf [20/06/2017].

SETH, C. (2013). La fabrique de l'intime. Mémoires et journaux de femmes du XVIII siècle. París: Robert Laffont. Collection Bouquins.

TROUSSON, R. (1996). Romans de femmes du XVIII siècle. París: Robert Laffont. Collection Bouquins.

VIENNOT, É. (2016). Et la modernité fut masculine. La France, les femmes et le pouvoir. 1789-1804. París: Perrin.

VIGÉE LE BRUN, É. (2008). Souvenirs, 1755-1842, G. Haroche-Bouzinac (ed.). París: Éditions Champion.

ZANONE, D. (2006). Écrire son temps. Les Mémoires en France de 1815 à 1848. Lyon: Presses Universitaires de Lyon.

Recibido el 2 de junio de 2017.

Aceptado el 15 de septiembre de 2017. 